Neurosurg Focus 18:(4):E12, 2005

\title{
Prognosis of gliomas in the 1970s and today
}

\author{
Joachim Oertel, M.D., Ph.D., Ekaterina von Buttlar, M.D., \\ Henry Werner Siegrried Schroeder, M.D., Ph.D., \\ and Michael Robert GaAb, M.D., Ph.D.
}

Department of Neurosurgery, Hannover Nordstadt Hospital, Hannover; and Department of
Neurosurgery, Ernst Moritz Arndt University, Greifswald, Germany

\begin{abstract}
The benefit of the current strategy for diagnosis (magnetic resonance [MR] imaging) and treatment (surgery, chemotherapy, radiotherapy) of gliomas, in contrast to the standard treatment in use before MR imaging and the microsurgical era, has not yet been determined. A retrospective statistical analysis was performed for all patients with glioma who underwent surgery at a single institution between 1965 and 1974 (Group I, 88 patients) or 1986 and 1995 (Group II, 249 patients). There were no major differences in symptomatology, tumor localization, and number of surgical procedures. The mean time until tumor diagnosis was significantly shorter in Group II (Group I, 48 weeks; Group II, 19.5 weeks). Also, the mean time from initial symptoms to surgery was significantly shorter for high-grade gliomas in Group II (Group I, 16.3 weeks; Group II, 11.7 weeks). For high- as well as low-grade gliomas, there was a clear reduction of the perioperative morbidity and mortality rates in Group II. Nevertheless, for the postoperative duration of survival, no significant differences were demonstrated for high- or low-grade gliomas. Based on the results of this study, the perioperative morbidity and mortality rate as well as the time from diagnosis to treatment have been remarkably reduced within the last 30 years. Nevertheless, the overall prognosis for patients with gliomas has not changed from the 1970s until today. Thus, the introduction of modern diagnostic modalities and surgical procedures has not improved the outcome in patients with glioma. Further research to improve the treatment of this disease is urgently needed.
\end{abstract}

KEY WORDS • astrocytoma • glioma • glioblastoma • diagnosis • prognosis •
neurosurgery

$\mathrm{A}$ $\mathrm{n}$ explosion in the development of new diagnostic modalities, surgical techniques, and adjuvant treatment strategies has changed the concept of glioma therapy remarkably in the last 30 years. The CT scanning introduced by Hounsfield in $1973^{1,10,11}$ and the nuclear MR imaging workers in 1978 and described in detail by Doyle, et al., in 1981 represent the "gold standard" for diagnosis and follow up of brain tumors. The use of the operating microscope has become a standard part of the technique in most intracranial procedures since the 1960s. ${ }^{17,32}$ Adjuvant therapy concepts, such as postoperative radiotherapy ${ }^{12}$ and nitrosourea chemotherapy $y^{7,8}$ became an additional treatment option in the 1960s and 1970s. Nevertheless, despite these remarkable advances, brain glioma remains a serious disease that is rarely cured. ${ }^{19}$ Although the mortality rate in patients with meningioma ${ }^{30}$ and in those with brain abscesses ${ }^{24}$ has been tremendously reduced since the pre-CT era, evidence of this is lacking for patients with intracranial gliomas.

Abbreviations used in this paper: $\mathrm{CT}=$ computerized tomography; KPS = Karnofsky Performance Scale; MR = magnetic resonance.
To evaluate what influence the introduction of the new diagnostic and therapeutic modalities had on the prognosis of patients with glioma as a distinct population, the authors retrospectively analyzed all patients who underwent operations for brain gliomas at a single institution in a 10-year period from 1965 to 1974 . The more recent 10year period from 1986 to 1995 served as a control. Special attention was paid to the time period required for diagnosis and subsequent surgery, initial symptomatology and tumor localization, applied diagnostic modalities, extent of surgery, applied adjuvant therapy, and prognosis as evaluated by postoperative survival duration and KPS score.

\section{Clinical Material and Methods}

\section{Patient Population and Inclusion Criteria}

A list of all patients with intracranial gliomas who underwent surgery at the Neurosurgical Department of the Ernst Moritz Arndt University in Greifswald, Germany between 1965 and 1974 (Group I) and between 1986 and 1995 (Group II) was derived from the hospital's surgical 

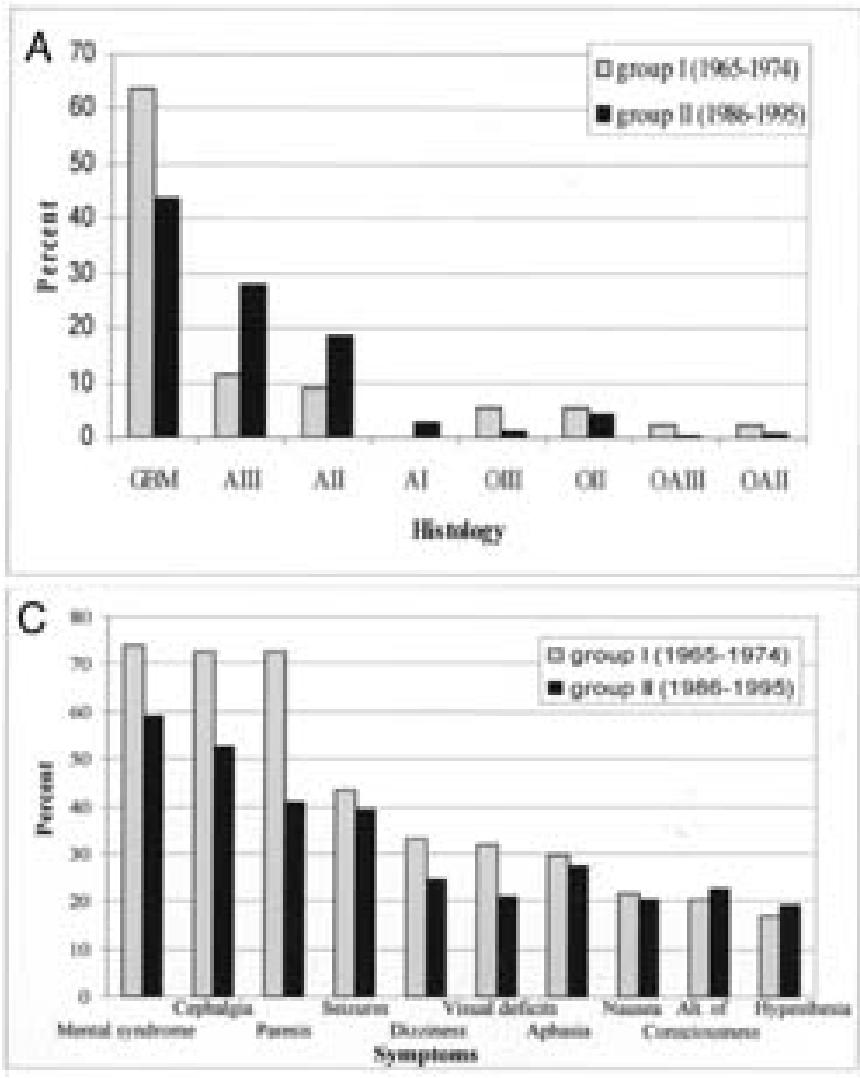

records. These time periods were selected because a 10year interval appeared appropriate to include a sufficient number of patients. In 1975, very few operations were performed due to reconstruction of the hospital. Thus, the period 1965 to 1974 was selected. To allow a direct comparison between times with and without MR imaging diagnostics, the second time period consisted of 1986 to 1995 , because an MR imaging unit was introduced at Greifswald in early 1991 after the reunification of Germany.

Inclusion criteria were as follows: minimum patient age of 18 years and histologically proven intracranial astrocytic, oligodendroglial, mixed glial tumor or glioblastoma according to the World Health Organization classification published by Kleihues, et al., in 1993. ${ }^{15,16}$ Patients with incomplete data sets regarding symptomatology, medical history, diagnostic modalities, type of surgery, applied adjuvant therapies, or pre- and postoperative functional status were excluded from the study.

\section{Data Evaluation}

Clinical data were obtained from the neurosurgical inpatient and postoperative follow-up records, and from documents of the referring institutions and other departments participating in the postoperative treatment as well as from the records of the general practitioners involved. Additionally, the time of death was determined through an inquiry at the National Cancer Registry (Gemeinsames Krebsregister, Berlin, Germany). The histological diagnosis was reevaluated according to the World Health Organization classification scheme from $1993^{15,16}$ and adjusted if indicated. Initial symptomatology, tumor loca-

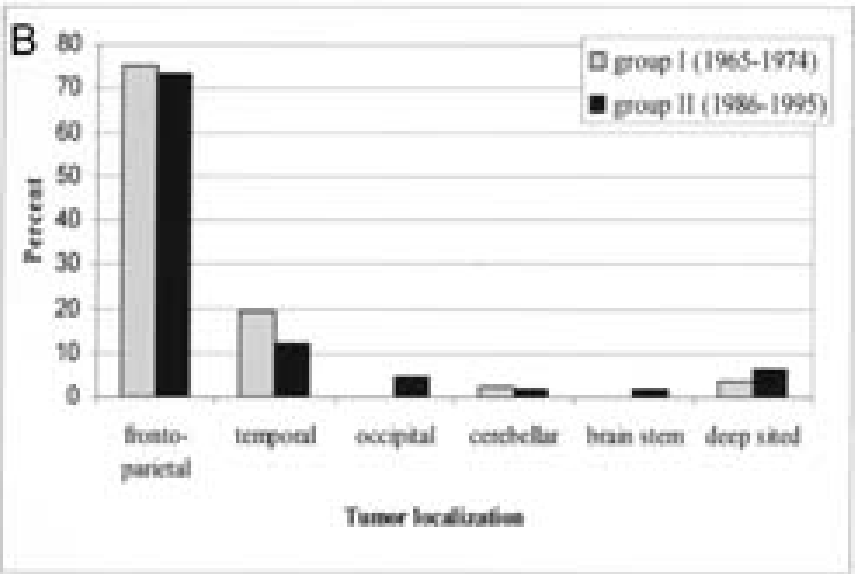

FIG. 1. Bar graphs showing the distribution of histological diagnoses (A), tumor localization (B), and symptomatology at initial presentation $(\mathrm{C})$. AI-III $=$ astrocytoma Grades I to III; Alt. = alteration; GBM = glioblastoma; OAII-III = oligoastrocytoma Grades II and III; OII-III = oligodendroglioma Grades II and III.

tion, kind of surgery, complications, tumor recurrence, and time of death were obtained. Special attention was given to the time interval from the onset of symptoms to diagnosis and surgical treatment, to the applied diagnostic procedures, to the application of the various adjuvant therapies, and to the postoperative duration of survival. The postoperative quality of life was assessed with the help of the KPS. A score of 70 or higher was defined as satisfactory quality of life. Because in a retrospective analysis the exact assessment of the KPS is almost impossible, the assessment was limited to a score of 70 or more or a score less than 70. This was evaluated at discharge, at 3 and 6 months, and at 1 year after surgery.

\section{Statistical Analysis}

The features of the two patient groups were compared using the Student two-sample t-test and the chi-square test. Paired t-tests were used to compare differences between values in the same group. Survival curves were calculated by the life table (Kaplan-Meier) method to account for varying periods of follow up.

\section{Results}

\section{Overall Results}

A total of 88 patients met the inclusion criteria in Group I (patients treated between 1965 and 1974), and a total of 249 patients were included in this study in Group II (those treated between 1986 and 1995). In both groups, more than $70 \%$ of all tumors consisted of glioblastomas or anaplastic astrocytomas (Fig. 1A) and were located in the 

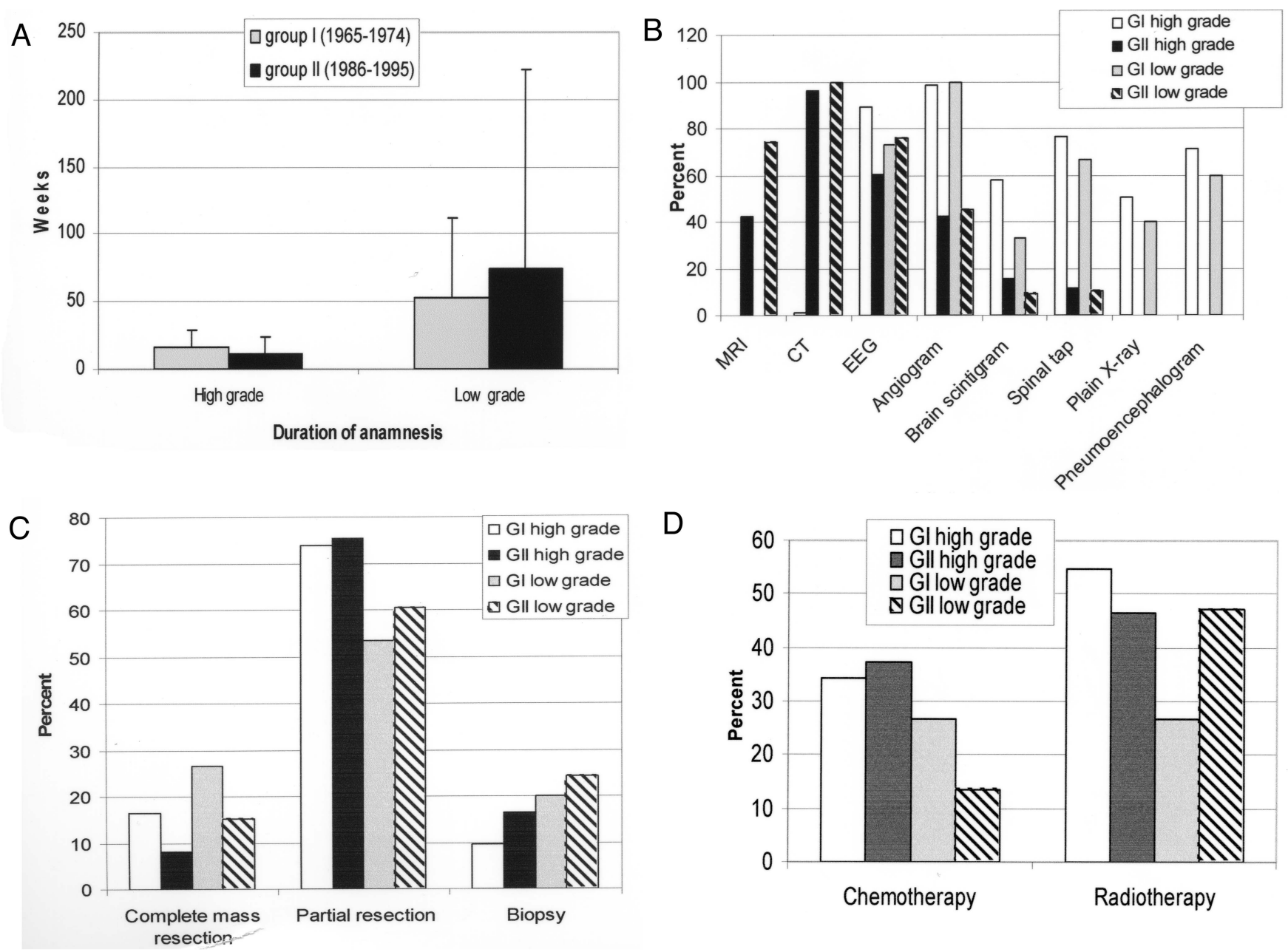

FIG. 2. Bar graphs showing time elapsed in weeks from initial symptoms to surgery (A). The duration from onset of symptoms for high-grade gliomas differed significantly according to the $\mathrm{t}$-test $(\mathrm{p}<0.01)$. Also shown are data for the application of various diagnostic modalities $(\mathrm{B})$, the frequency of the various surgical procedures $(\mathrm{C})$, and the frequency of postoperative adjuvant therapies (D). EEG = electroencephalography; GI = Group I; GII = Group II.

frontoparietal lobes (Fig. 1B). In both groups, mental syndrome and headache, followed by paresis and seizures, were the most common symptoms at initial presentation (Fig. 1C).

\section{High-Grade Gliomas}

In 73 patients in Group I and 183 in Group II, the histological diagnosis of their tumors was Grade III or Grade IV glioma. The mean age of patients with high-grade gliomas was 45.7 years (range 21-69 years) in Group I and 51.7 years (range 18-73 years) in Group II. Group I consisted of $69.9 \%$ men, and Group II was made up of $56.3 \%$ men.

\section{Duration of Symptoms}

The time from initial (most likely specific) symptoms (paresis, hypesthesia, seizures corresponding to the localization of the tumor) to surgery was $16.33 \pm 13.1$ weeks in Group I and $11.7 \pm 11.6$ weeks in Group II (Fig. 2A). This difference was highly significant according to the ttest $(\mathrm{p}<0.01)$.

\section{Diagnostic and Surgical Procedures, Adjuvant Therapies}

The frequency with which the diagnostic modalities were used is given in Fig. 2B. The differences between Groups I and II were significant according to the chisquare test $(\mathrm{p}<0.05)$.

A partial resection of the tumor mass was the most frequent procedure in both groups, with $74 \%$ in Group I and $75.4 \%$ in Group II (Fig. 2C). An open biopsy procedure was performed in $9.6 \%$ of patients in Group I and $16.4 \%$ in Group II, and a complete resection of the tumor mass was accomplished in $16.4 \%$ of the patients in Group I and in $8.2 \%$ in Group II. Adjuvant therapies such as postoperative chemotherapy and radiation treatment were used with similar frequency in both groups (Fig. 2D). There were no significant differences according to the chi-square test. In eight patients (11\%) in Group I and in $23(12.6 \%)$ in Group II, subsequent surgery for recurrent tumor growth was performed.

\section{Perioperative Morbidity and Mortality Rates}

There was no significant difference in the number of 

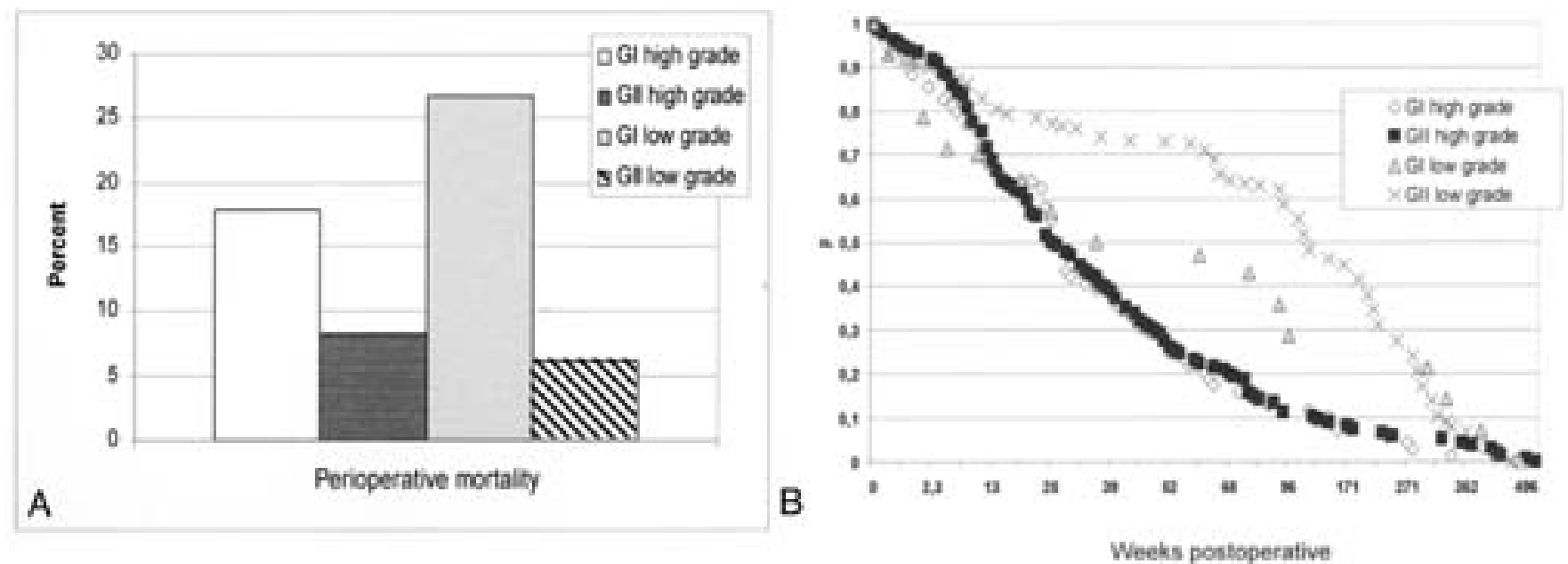

FIG. 3. A: Bar graph showing mortality levels within 4 weeks postsurgery. There was a statistically significant reduction of the mortality level for high- and low-grade gliomas between Group I and Group II. B: Kaplan-Meier curves showing postoperative duration of survival for low- and high-grade gliomas. $p=$ percentages given as decimels.

perioperative surgical complications, with $15 \%$ in Group I and $13.6 \%$ in Group II, as shown in Table 1. Nevertheless, with respect to the perioperative mortality (defined as death within 4 weeks postsurgery) there was a statistically significant reduction, from $17.8 \%$ in Group I to $8.2 \%$ in Group II (Fig. 3A).

\section{Postoperative Duration of Survival and Quality of Life}

In Group I 82.2\% of the patients and in Group II 91.8\% survived at 4 weeks postsurgery. At 6 months postsurgery, $56 \%$ of Group I and $55.1 \%$ of Group II patients remained alive. At 2 years postsurgery, 14.1 and $17 \%$ of patients in Groups I and II, respectively, survived. Data are shown according to the Kaplan-Meier method (Fig. 3B). The slight differences observed were not statistically significant.

At discharge, $71.2 \%$ of the patients in Group I and $68.7 \%$ of those in Group II had a KPS score of 70 or higher. At 3, 6, and 12 months postsurgery, this percentage was $58.5,63.2$, and $66.6 \%$, respectively, for Group I and 75.9, 71.4 , and $73.8 \%$, respectively, in Group II. In all, the number of patients alive with a KPS score of 70 or higher was slightly larger in Group II at 3, 6, and 12 months postsurgery; however, this difference did not reach significance according to the chi-square test.

\section{Low-Grade Gliomas}

Gliomas in 15 patients in Group I and 66 in Group II were histologically diagnosed as Grade I or Grade II. The mean age for patients with low-grade gliomas was 39.7 years (range 24-59 years) in Group I and 42.2 years (range 18-65 years) in Group II. Group I consisted of $53.3 \%$ men, and Group II was made up of $40.9 \%$ men.

\section{Duration of Symptoms}

The time from initial (most likely specific) symptoms (paresis, hypesthesia, seizures corresponding to the localization of the tumor) to surgery was $52.7 \pm 59$ weeks in Group I and 74.2 \pm 147.8 weeks in Group II, with median values of 35.6 and 19 weeks, respectively (Fig. 2A).
There was no significant difference between both groups.

\section{Diagnostic and Surgical Procedures, Adjuvant Therapies}

The frequency with which the diagnostic modalities were used is given in Fig. 2B. According to the chi-square test, the differences between Groups I and II were significant for all modalities except electroencephalography and brain scintigraphy $(\mathrm{p}<0.05)$.

Again, a partial tumor resection was the most frequent procedure in both groups; $53.3 \%$ in Group I and 60.6\% in Group II (Fig. 2C). An open biopsy was performed in $20 \%$ of patients in Group I and in $24.2 \%$ in Group II, and complete resection of the tumor mass was accomplished in $26.7 \%$ of the patients in Group I and in $15.2 \%$ in Group II. Adjuvant therapies were used with similar frequency in both groups, with a tendency toward more frequent application of radiotherapy and less frequent application of chemotherapy in Group II (Fig. 2D). There were no significant differences according to the chi-square test.

In four patients $(33.3 \%)$ in Group I and $16(24.2 \%)$ in Group II, subsequent surgery for recurrent tumor growth was performed.

\section{Perioperative Morbidity and Mortality Rates}

There was a clear difference in the number of perioperative surgical complications, with $20.1 \%$ in Group I and $4.5 \%$ in Group II, as shown in Table 1. Nevertheless, this difference did not reach statistical significance according to the Fisher test $(p=0.07)$. With respect to perioperative mortality, defined as death within 4 weeks postsurgery, there was a statistically significant reduction from $26.7 \%$ in Group I to $6.1 \%$ in Group II (Fig. 3A).

\section{Postoperative Duration of Survival and Quality of Life}

In Group I 73.3\% of the patients and in Group II 93.9\% survived at 4 weeks postsurgery. At 6 months postsurgery, $57.1 \%$ of Group I and $79.3 \%$ of Group II patients remained alive. At 1 and 2 years postsurgery, 50 and $75.9 \%$, respectively, in Group I as well as 28.6 and $55.2 \%$ of the patients in Group II survived. As demonstrated in Fig. 3B, 
TABLE 1

Complications in patients with gliomas treated before and after the advent of CT and MR imaging*

\begin{tabular}{|c|c|c|c|c|}
\hline \multirow[b]{2}{*}{ Complication } & \multicolumn{2}{|c|}{$\begin{array}{l}\text { High-Grade } \\
\text { Gliomas (\%) }\end{array}$} & \multicolumn{2}{|c|}{$\begin{array}{l}\text { Low-Grade } \\
\text { Gliomas (\%) }\end{array}$} \\
\hline & Group I & Group II & Group I & Group II \\
\hline hydrocephalus & $1(1.4)$ & $0(0.0)$ & $0(0.0)$ & $1(1.5)$ \\
\hline intracerebral hematoma & $2(2.7)$ & $7(3.8)$ & $1(6.7)$ & $0(0.0)$ \\
\hline superficial wound infection & $3(4.1)$ & $7(3.8)$ & $0(0.0)$ & $0(0.0)$ \\
\hline brain abscess formation/meningitis & $2(2.7)$ & $3(1.6)$ & $1(6.7)$ & $0(0.0)$ \\
\hline postop brain edema & $3(4.1)$ & $8(4.4)$ & $1(6.7)$ & $2(3)$ \\
\hline total & $11(15.0)$ & $25(13.6)$ & $3(20.1)$ & $3(4.5)$ \\
\hline
\end{tabular}

* Group I consisted of patients treated between 1965 and 1974, and Group II contained patients treated between 1986 and 1995.

there was a much higher percentage of surviving patients in Group II up to 4 years postsurgery. Nevertheless, this difference did not reach statistical significance at 25, 50, 100 , and 150 weeks postsurgery.

At discharge, $66.7 \%$ of the patients in Group I and $87.1 \%$ of those in Group II had a KPS score of 70 or higher. At 3, 6, and 12 months postsurgery, this percentage was $95.4,100$, and $100 \%$, respectively, for Group I and 87.1, 85.3 , and $84.4 \%$, respectively, in Group II. The percentage of patients alive with a KPS score of 70 or higher was not significantly different between the groups.

\section{Discussion}

Although there have been notable technical advances in the diagnosis of and the surgical approach to brain tumors, including CT and MR imaging ${ }^{5,28}$ for diagnosis and stereotactic surgery, intraoperative cortical sensory and motor mapping, computer-assisted laser resection, and neuronavigation for surgery, $, 13,14,20,31$ the consequences of these new modalities on the extent of resection and on the prognosis of patients with glioma remain under intense discussion. ${ }^{21,29}$ The goal of our study was to evaluate whether changes in diagnostic modalities and surgical procedures within the last 30 years has indeed had an impact on the prognosis of patients with glioma in a distinct patient population. In this study we report results in 337 patients with gliomas who underwent surgery either between 1965 and 1974 or between 1986 and 1995 at a single institution.

Based on the data in this study, the time between initial symptoms and surgery has been reduced for malignant as well as benign glial tumors from the 1970s to the mid1990s. Most likely, this can be attributed to the ubiquitous availability of newly introduced diagnostic methods such as $\mathrm{CT}$ and MR imaging. In addition, the reduced rates of perioperative morbidity and mortality (see next paragraph) most likely also aids the decision to perform surgery, and more and more patients with low-grade gliomas undergo tumor resection.

In addition to the skill and experience of the surgeon, there are several factors that affect the incidence of complications in brain tumor surgery. Variables such as tumor location, extent of resection, previous treatment, tumor characteristics (size and histological features), the patients' preoperative neurological and physical status, age, and the availability of monitoring and intraoperative navigational devices can all influence the surgical out- come. ${ }^{3,25,27}$ In our study, a significant reduction in perioperative mortality was found for high-grade as well as lowgrade gliomas in Group II. Also, the number of perioperative complications was smaller in Group II for all gliomas, although this difference did not reach statistical significance. Thus, modern diagnostic and surgical procedures have most likely contributed to the reduction of the perioperative morbidity and mortality rates in gliomas.

In this study we failed to demonstrate any significant difference in the long-term survival for high-grade or for low-grade gliomas. Historically, the median duration of survival has been less than 1 year in patients with glioblastoma multiforme ${ }^{26}$ and less than 2 years in those with anaplastic astrocytomas. ${ }^{2}$ For low-grade gliomas, exact data are missing because the diagnosis is often made incidentally. Nevertheless, particularly for oligodendrogliomas, some authors report significantly better patient survival with the use of diagnostic CT scanning than was seen before this modality was available. In 1994, Celli, et al., ${ }^{4}$ reported significantly better survival rate for patients treated in the modern era (1977-1986), when CT scanning was available, compared with those treated before. Shimizu, et al., ${ }^{28}$ demonstrated a 5-year survival rate of $76 \%$ in patients who underwent CT scanning compared with $41 \%$ in the group of patients who did not. These data indicate that patients with oligodendrogliomas diagnosed in the CT/MR imaging era may have a longer survival duration than those who underwent surgery earlier. In contrast, Leonardi and Lumenta ${ }^{18}$ concluded from their data that, despite new diagnostic and therapeutic tools, no prolongation of survival duration for oligodendrogliomas was seen in the CT/MR imaging era. Our study supports the latter observation because no improvement of the longterm survival duration was demonstrated. Nevertheless, particularly for low-grade gliomas, the number of patients in Group I is very small. Also, a trend toward better survival of these patients in Group II is notable. Thus, no final conclusion can be drawn from the present data for low-grade gliomas.

In all, our study demonstrates that the optimal treatment of gliomas still has not been discovered. Since the introduction of the current standard diagnostic modalities such as $\mathrm{CT}$ and MR imaging and surgical procedures such as neuronavigation and microsurgery, no great impact of these changes on the prognosis of gliomas could be found. Nevertheless, the time from initial symptoms to surgery and the perioperative morbidity and mortality rates have clearly been reduced since the 1970s. Further research to find the optimal therapy for gliomas has to be conducted. The further development of new surgical procedures could contribute to improvement in glioma prognosis ${ }^{9,22,23}$ Attempts to prolong the median survival duration of cancer patients also must focus on developing new chemotherapeutic agents, radiotherapy methods, and immunotherapies.

\section{References}

1. Ambrose J: Computerized x-ray scanning of the brain. J Neurosurg 40:679-695, 1974

2. Burger PC, Green SB: Patient age, histological features, and length of survival in patients with glioblastoma multiforme. Cancer 59:1617-1625, 1987 
3. Cabantong AM, Bernstein M: Complications of first craniotomy for intra-axial brain tumour. Can J Neurol Sci 21:213-218, 1994

4. Celli P, Nofrone I, Palma L, et al: Cerebral oligodendroglioma: prognostic factors and life history. Neurosurgery 35: 1018-1035, 1994

5. Coffey RJ, Lunsford LD, Taylor FH: Survival after stereotactic biopsy of malignant gliomas. Neurosurgery 22:465-473, 1988

6. Doyle FH, Gore JC, Pennock JM, et al: Imaging of the brain by nuclear magnetic resonance. Lancet 2:53-57, 1981

7. Fewer D, Wilson CB, Boldrey EB, et al: The chemotherapy of brain tumors. Clinical experience with carmustine (BCNU) and vincristine. JAMA 222:549-552, 1972

8. Hochberg FH, Linggood R, Wolfson L, et al: Quality and duration of survival in glioblastoma multiforme. Combined surgical, radiation and lomustine therapy. JAMA 241:1016-1018, 1979

9. Höllerhage HG; Zumkeller M, Becker M, et al: Influence of type and extent of surgery on early results and survival time in glioblastoma multiforme. Acta Neurochir 113:31-37, 1991

10. Hounsfield GN: Computed medical imaging. Science 210: 22-28, 1980

11. Hounsfield GN: Computerized transverse axial scanning (tomography). I. Description of system. Br J Radiol 46: 1016-1022, 1973

12. Jelsma R, Bucy PC: The treatment of glioblastoma multiforme of the brain. J Neurosurg 27:388-400, 1967

13. Kelly PJ: Stereotactic biopsy and resection of thalamic astrocytomas. Neurosurgery 25:185-195, 1989

14. Kelly PJ, Daumas-Duport C, Scheithauer BW, et al: Stereotactic histologic correlation of computed tomography and magnetic resonance imaging-defined abnormalities in patients with glial neoplasms. Mayo Clin Proc 62:450-459, 1987

15. Kleihues P, Burger PC, Scheithauer BW: Histological typing of tumours of the central nervous system, ed 2. Berlin: Springer-Verlag, 1993

16. Kleihues P, Burger PC, Scheithauer BW: The new WHO classification of brain tumours. Brain Pathol 3:255-268, 1993

17. Kriss TC, Kriss VM: History of the operating microscope: from magnifying glass to microneurosurgery. Neurosurgery 42: 899-908, 1998

18. Leonardi MA, Lumenta CB: Oligodendrogliomas in the CT/MR-era. Acta Neurochir 143:1195-1203, 2001

19. Mahaley MS, Mettlin C, Natarajan N, et al: National survey of patterns of care for brain-tumor patients. J Neurosurg 71: 826-836, 1989
20. Marshall LF, Jennett B, Langfitt TW: Needle biopsy for the diagnosis of malignant glioma. JAMA 228:1417-1418, 1974

21. Nazzaro JM, Neuwelt EA: The role of surgery in the management of supratentorial intermediate and high-grade astrocytomas in adults. J Neurosurg 73:331-344, 1990

22. Oertel J, Gaab MR, Knapp A, et al: Water jet dissection in neurosurgery: experimental results in the porcine cadaveric brain. Neurosurgery 52:153-159, 2003

23. Oertel J, Gaab MR, Pillich DT, et al: Comparison of waterjet dissection and ultrasonic aspiration: an in vivo study in the rabbit brain. J Neurosurg 100:498-504, 2004

24. Rosenblum ML, Hoff JT, Norman D, et al: Decreased mortality from brain abscesses since advent of computerized tomography. J Neurosurg 49:658-668, 1978

25. Salcman M: Surgical decision-making for malginant brain tumors. Clin Neurosurg 35:285-313, 1989

26. Salcman M: Survival in glioblastomas: historical perspective. Neurosurgery 7:435-439, 1980

27. Sawaya R, Rambo WM Jr, Hammoud MA, et al: Advances in surgery for brain tumors. Neurol Clin 13:757-771, 1995

28. Shimizu KT, Tran LM, Mark RJ, et al: Management of oligodendrogliomas. Radiology 186:569-572, 1993

29. Stummer W, Novotny A, Stepp H, et al: Fluorescence-guided resection of glioblastoma multiforme by using 5-aminolevulinic acid-induced porphyrins: a prospective study in 52 consecutive patients. J Neurosurg 93:1003-1013, 2000

30. Turgut M, Ozcan OE, Benli K, et al: Factors affecting morbidity and mortality following surgical intervention in patients with intracranial meningioma. Aust N Z J Surg 66:144-150, 1996

31. Wagner W, Gaab MR, Schroeder HWS, et al: Cranial neuronavigation in neurosurgery: assessment of usefullness in relation to type and site of pathology. Minim Invasive Neurosurg 43: $124-131,2000$

32. Yaşargil MG: A legacy of microneurosurgery: memoirs, lessons, and axioms. Neurosurgery 45:1025-1092, 1999

Manuscript received March 30, 2005.

Accepted in final form April 6, 2005.

Parts of this study were supported by a grant from the Else Kröner Fresenius Stiftung, Bad Homburg, Germany.

Address reprint requests to: Joachim Oertel, M.D., Ph.D., Department of Neurosurgery, Hannover Nordstadt Hospital, Haltenhoffstrasse 41, 30167 Hannover, Germany. email: oertelj@ freenet.de. 